\title{
Predictors of discontinuation, efficacy, and safety of memantine treatment for Alzheimer's disease: meta-analysis and meta-regression of 18 randomized clinical trials involving 5004 patients
}

Lídia Blanco-Silvente ${ }^{1,2^{*}}$ D. Dolors Capellà ${ }^{1,2}$, Josep Garre-Olmo ${ }^{2,3}$, Joan Vilalta-Franch ${ }^{2,3}$ and Xavier Castells ${ }^{1,2}$

\begin{abstract}
Background: The risk-benefit relationship of memantine treatment for Alzheimer's disease (AD) remains unclear. In addition, variability between the results of clinical trials has been observed. The aim of this study was to investigate the risk-benefit relationship of memantine treatment in patients with $A D$ and to determine the predictor effect of patient, intervention, and study design related covariates.

Methods: A systematic review and meta-analysis of double-blind, placebo controlled clinical trials was performed. Primary outcomes were all-cause discontinuation, discontinuation due to adverse events (AE) and efficacy on cognitive function. Odds ratio (OR) and standard mean difference (SMD) with 95\% confidence intervals were calculated. Metaregression was conducted to identify related covariates. Cochrane Collaboration tool was used to evaluate the risk of bias of included trials.

Results: Eighteen studies involving 5004 patients were included. No differences between memantine and placebo were found for all-cause treatment discontinuation $(\mathrm{OR}=0.97[0.82,1.14])$ and discontinuation due to $A E(O R=1.18[0.91,1.53])$. Memantine showed small improvement on cognitive function (SMD=0.15 [0.08, 0.22]). Baseline functional ability was positively associated with all-cause treatment discontinuation and discontinuation due to $\mathrm{AE}$.

Conclusions: Our study suggests that memantine has a very small efficacy on AD symptomatology and its safety profile is similar to that of placebo. No evidence of treatment discontinuation improvement with memantine is found, indicating a dubious risk-benefit relationship. No intervention characteristic or subgroup of patients clearly shows a significantly better risk-benefit relationship.
\end{abstract}

PROSPERO registration: CRD42014015696.

Keywords: Alzheimer's disease, Memantine, Discontinuation, Efficacy, Meta-analysis, Meta-regression

\footnotetext{
* Correspondence: lidia.blanco@udg.edu

${ }^{1}$ TransLab Research Group; Department of Medical Sciences, University of Girona, Girona, Spain

${ }^{2}$ Department of Medical Sciences, Faculty of Medicine, University of Girona,

Emili Grahit, 77, 17003 Girona, Spain

Full list of author information is available at the end of the article
}

(c) The Author(s). 2018 Open Access This article is distributed under the terms of the Creative Commons Attribution 4.0 International License (http://creativecommons.org/licenses/by/4.0/), which permits unrestricted use, distribution, and reproduction in any medium, provided you give appropriate credit to the original author(s) and the source, provide a link to the Creative Commons license, and indicate if changes were made. The Creative Commons Public Domain Dedication waiver (http://creativecommons.org/publicdomain/zero/1.0/) applies to the data made available in this article, unless otherwise stated. 


\section{Background}

Around 47 million people worldwide have dementia and, by 2030 , it is expected to affect 75.6 million people, with Alzheimer's disease (AD) being the most common cause [1]. AD is a neurodegenerative disorder characterised by cognitive impairment, behaviour disturbances and functional disability. AD incurs significant health and community care costs [2], and both cognitive and functional decline are associated with caregiver outcomes [3].

Memantine is a non-competitive N-methyl-D-aspartate (NMDA) receptor antagonist that has shown a neuroprotective effect in some studies $[4,5]$. It is approved for the treatment of moderate to severe $\mathrm{AD}[6,7]$, and some guidelines support using it in combination with a cholinesterase inhibitor (ChEI) [8] whereas others do not recommend it because important gaps in the evidence exist [9]. Since memantine is the only drug authorized for treating patients with moderate-severe $\mathrm{AD}$, its frequent prescription is unsurprising [10-13]. Nevertheless, the risk-benefit relationship associated with it remains unclear for several reasons. Firstly, the efficacy of memantine has been studied fundamentally on the severity of AD symptoms. However, the use of this outcome is arguable because of its dubious clinical relevance. For this reason, pragmatic outcomes like institutionalisation or mortality have been recommended [9]. In addition, the validity of 'symptom improvement' may be hampered by blinding failure and attrition bias [14]. Secondly, memantine has been associated with several side effects such as dizziness, headaches, constipation, somnolence, hypertension and agitation, some of which may be serious $[6,7$, 13]. In this context, it is difficult to weigh memantine's efficacy against its safety. This problem can be partly overcome using "all-cause treatment discontinuation", a pragmatic outcome that reflects therapeutic benefits in relation to undesirable effects [15]. In addition, it is unaffected by attrition bias as this outcome has no missing data. Treatment discontinuation has been used previously in the field of $\mathrm{AD}$ [15-17] and other disorders [18-20].

Another issue that further complicates the assessment of memantine's risk-benefit relationship is the variability of results between different clinical trials. While some studies show positive findings on cognitive symptoms [21, 22] and discontinuation rate, others find no differences from placebo [23-27]. Between-study variability can be determined by means of meta-analysis, which allows for calculating the proportion of the variability in effect estimates that is due to heterogeneity rather than chance [28]. The presence of statistical heterogeneity reflects inconsistency, and this reduces the confidence in the meta-analysis findings and the strength of clinical recommendations derived from them. Determining the sources of between-study variability enables clustering the studies in groups with more consistent results for which specific clinical recommendations should be made [29]. Meta-regression can be used to determine the sources of between-study variability, which could be related to the following: (1) the study design, such as whether there is a lead-in period [30] and the number of study sites [31]; (2) the intervention studied, for example dose [32, 33] and length of treatment [34, 35]; (3) patient characteristics, for example age [36] and severity of the disease [37]; and (4) study sponsorship [19, 38]. Identifying these factors can help tailor treatment with memantine for patients with $A D$ and guide future research.

Previous meta-analyses have analysed the efficacy and safety of memantine for AD [17, 39-48]; however, none has used all-cause discontinuation to assess the risk-benefit treatment relationship, nor has investigated extensively the sources of between-study variability in different outcomes of discontinuation, efficacy, and safety. To fill this gap, this study aims to (1) evaluate the risk-benefit relationship of memantine in patients with $\mathrm{AD}$ using all-cause treatment discontinuation as the primary outcome; (2) determine the predictor effect of study design, patient, and intervention related covariates on discontinuation, efficacy, and safety outcomes by performing meta-analysis and meta-regression.

\section{Methods}

\section{Study design and search strategy}

We conducted a systematic review and meta-analysis (SRMA) of double-blind, randomized, placebo-controlled, parallel-group clinical trials (RPCCT) that investigated the effect of memantine (dose 5 to $23 \mathrm{mg} /$ day) in monotherapy, or in combination with ChEI in AD patients. The length of intervention was at least 12 weeks. We excluded articles in languages other than English, Spanish, Italian, French and Portuguese and studies published only as conference abstracts. The study protocol was registered at the International Prospective Register of Systematic Reviews (PROSPERO): CRD42014015696. The Preferred Reporting Items for Systematic Reviews and Meta-analysis (PRISMA) guidelines [49] were followed (see Additional file 1: Table S1).

The following databases were searched to identify studies meeting our inclusion criteria: Medline, Cochrane Central Register of Controlled Trials, PsycINFO, ISI Web of Knowledge, www.clinicaltrials.gov, www.clinicaltrialsregister.eu, www.controlled-trials.com. The search strategy is detailed in the (Additional file 1: Table S2). Reference lists of previous systematic reviews [17, 39-48], pharmaceutical industry databases and reports from drug regulatory agencies such as European Medicines Agency and Food and Drug Administration were reviewed to identify further studies. The limit of the search was 01 February 2017. 


\section{Data extraction and quality assessment}

Data extraction was carried out independently by two reviewers $(\mathrm{LB}, \mathrm{XC})$ and disagreements were discussed with a third reviewer (DC). Study authors and pharmaceutical companies were emailed to obtain unpublished data. The risk of bias of the RPCCT included was assessed using the Cochrane Collaboration tool. This tool evaluates the risk of bias based on the description and suitability of the following domains: sequence generation, allocation concealment, blinding, incomplete data, selective outcome reporting, and other biases. A judgement relating to the risk of bias is given for each domain in terms of 'low,' 'high', or 'unclear' risk.

\section{Study outcomes}

The primary outcomes were 1) all-cause treatment discontinuation, defined as the proportion of patients who did not complete the study for some reason; 2) discontinuation due to adverse events (AE), defined as the proportion of patients who dropped out due to side effects; and 3) efficacy on cognitive function, defined as the improvement in cognitive symptoms, giving preference to the Alzheimer's disease Assessment Scale-Cognitive subscale (ADAS-cog) [50], followed by Mini-Mental State Examination (MMSE) [51], and then the Severe Impairment Battery (SIB) [52].

The secondary outcomes were 1) discontinuation due to lack of efficacy (LoE), defined as the proportion of patients who did not complete the study for inefficacy; 2) efficacy on global change from baseline, giving priority to the Clinician Interview-Based Impression on Change-Plus Caregiver Input (CIBIC-Plus) [53] over the Clinical Global Impression (CGI) [54]; 3) efficacy on neuropsychiatric symptoms, with preference given to the Neuropsychiatric Inventory (NPI) [55] over the Behavioural Pathology in Alzheimer's Disease Rating Scale (BEHAVE-AD) [56]; 4) efficacy on functional ability, giving preference to the Alzheimer's Disease Cooperative Study Activities of Daily Living Inventory 19- or 23-item Scale (ADCS-ADL) [57] over the Disability Assessment for Dementia (DAD) [58]; 5) mortality, as the proportion of patients who died; 6) AE, defined as the proportion of patients experiencing any side effect; 7) serious adverse events (SAE), defined as the proportion of patients experiencing one or more SAE and 8) drug-related adverse event (DRAE), defined as the proportion of patients experiencing one or more $\mathrm{AE}$ considered related with drug intervention by the investigator during the study. We preferred intention to treat analysis data (ITT) to per-protocol (PP). For efficacy outcomes, we preferred changes scores to endpoint scores, and these to response rates.

The following covariates were collected: number of study sites (single vs multi-site); lead-in period (yes vs no); placebo lead-in period (yes vs no); type of statistical analysis
(ITT vs non-ITT); memantine intervention (monotherapy vs combination with $\mathrm{ChEI}$ ); dose (20 vs $28 \mathrm{mg} /$ day); regimen (qd vs bid); dosage (fixed vs flexible); length of the intervention (weeks); age (years); gender (\% women); AD baseline severity (mild, mild-moderate, moderate, moderate-severe, severe); baseline cognitive function (\% scale maxima); baseline neuropsychiatric symptom severity (\% scale maxima); baseline functional ability (\% scale maxima) and study funding (independent vs industry). Regarding the type of statistical analysis carried out, we considered ITT approach when the number of patients included in the efficacy analyses was at least $95 \%$ of the total number of randomized patients, the others being non-ITT. Regarding memantine intervention, we considered that memantine was administered in combination with ChEI when more than $50 \%$ of patients received donepezil, galantamine or rivastigmine.

Baseline cognitive function, neuropsychiatric symptoms and functional ability were assessed using various scales. In order to standardize the baseline scores of these covariates, we calculated the percentage of scale maxima, which re-expresses the score as if the scale ranged from 0 to 100 .

\section{Statistical analysis}

Odds ratio (OR) and 95\% confidence intervals (CI) were calculated for dichotomous outcomes, and standardized mean difference (SMD) for continuous ones. For efficacy outcomes we used change scores, endpoint scores, and response rates as their combination has been shown to be valid $[59,60]$. OR were re-expressed as SMD to allow them to be combined with continuous outcomes [61] (For an example on how we combined different efficacy scales and scores see Additional file 2: Table S3). A SMD of 0.2 was considered small, of 0.5 moderate, and SMD above 0.8 was considered large [62]. For outcomes where the efficacy was assessed using the same rating scale, mean difference (MD) was calculated. In RPCCT that compared memantine in monotherapy and in combination with ChEI vs. placebo, we analysed the effect of each intervention separately. However, the number of patients in the placebo group was divided by two to avoid over-counting [60]. Heterogeneity was assessed using the uncertainty factor $I^{2}$, which measures the percentage of variance across studies that is due to heterogeneity rather than chance [62]. We combined OR and SMD by means of a random effects model [63], which takes into account both within- and between-study heterogeneity. The potential sources of heterogeneity were analysed using meta-regression [64] irrespective of the percentage of $I^{2}$, because of the low sensitivity of the test. To quantify the proportion of variance explained by the covariate, we calculated the $\mathrm{R}^{2}$ index, which represents the ratio of explained variance to total variance 
[65]. All analyses were conducted using Comprehensive Meta-Analysis software (version 3) [66]. The full study dataset is provided in the (Additional file 2: Tables S4-S13).

Two sensitivity analyses were performed by 1) repeating the analysis after the exclusion of RPCCT with a high risk of bias in at least one domain; and 2) including the results of one pooled analysis [67], which reports the results of two Japanese clinical trials whose primary results could not be found (post-hoc analysis). Publication bias was assessed with funnel plots [68] and Begg's [69] and Egger's test [70].

\section{Results}

\section{Study design, intervention and patient characteristics}

Eighteen studies were included (see Fig. 1 and Additional file 3: Table S14 and Table S15) involving nineteen memantine vs placebo comparisons. Table 1 shows study design, intervention and patients' characteristics. Most studies were multi-site (77.8\%), one third (33.3\%) had a placebo lead-in period, and a high proportion of them $(83.3 \%)$ had commercial sponsorship. Slightly over half (55.6\%) of the studies included patients with moderate-severe AD. ITT was the most common statistical approach, except in neuropsychiatric symptoms, in which case non-ITT analysis prevailed (85.7\%) (see Additional file 3: Table S16).

Regarding interventions, memantine in monotherapy was investigated in 11 studies and in combination with ChEI in 8 studies. One trial [71] had a factorial design and studied memantine and donepezil both alone and combined. All studies but one [21] investigated $20 \mathrm{mg} /$ day of memantine in a fixed dosage. The majority of studies investigating memantine in monotherapy used a twice daily regimen $(81.8 \%)$, whereas a once daily regimen was more frequent in studies investigating memantine in combination with ChEI (62.5\%). The length of the intervention ranged from 12 to 208 weeks, with a

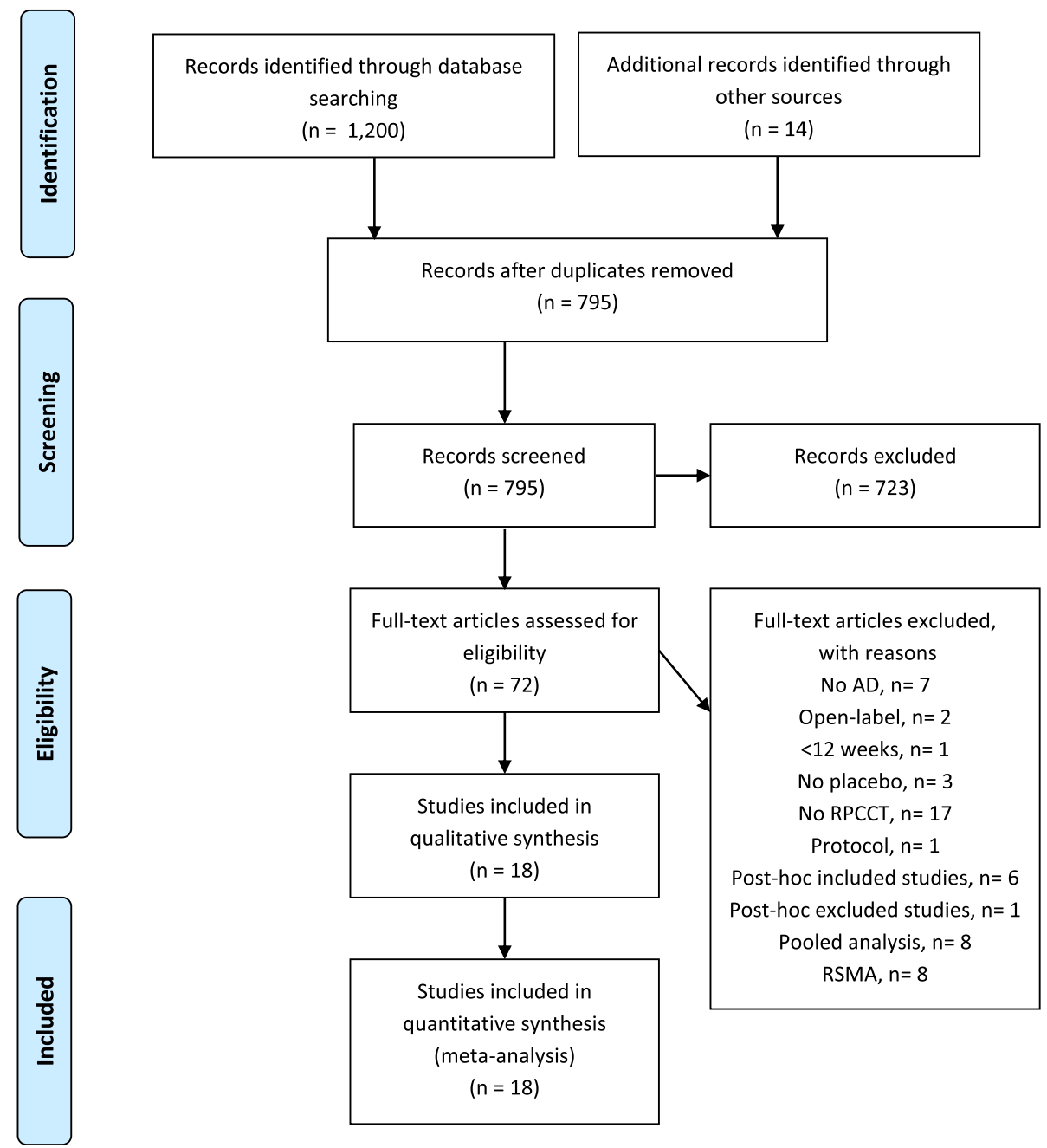

Fig. 1 Preferred Reporting Items for Systematic Reviews and Meta-analyses (PRISMA) flow diagram 
Table 1 Studies, interventions and patients' characteristics, and risk of bias of included clinical trials

\begin{tabular}{|c|c|}
\hline \multicolumn{2}{|l|}{ Studies } \\
\hline Number of studies ${ }^{a}$ & 18 \\
\hline Number of drug-placebo comparisons & 19 \\
\hline Number of patients/study (median) [range] & 287 [13-677] \\
\hline Multi-site studies (\%) & 77.8 \\
\hline Lead-in period (\%) & 33.3 \\
\hline Placebo lead-in period (\%) & 100 \\
\hline \multicolumn{2}{|l|}{ AD severity (\%) } \\
\hline Mild & 0 \\
\hline Mild-moderate & 33.3 \\
\hline Moderate & 11.1 \\
\hline Moderate-severe & 55.6 \\
\hline Severe & 0 \\
\hline \multicolumn{2}{|l|}{ Study funding (\%) } \\
\hline Independent & 16.7 \\
\hline Industry & 83.3 \\
\hline \multicolumn{2}{|l|}{ ITT statistical analysis (\%) } \\
\hline Discontinuation outcomes & 100 \\
\hline Efficacy cognitive function & 56.2 \\
\hline Efficacy global change & 70.0 \\
\hline Efficacy neuropsychiatric symptoms & 14.3 \\
\hline Efficacy functional ability & 60.0 \\
\hline Safety outcomes & 100 \\
\hline \multicolumn{2}{|l|}{ Intervention ${ }^{\mathrm{b}}$} \\
\hline Monotherapy (\%) & 57.9 \\
\hline Combination ChEl (\%) & 42.1 \\
\hline \multicolumn{2}{|l|}{ Dose (\%) } \\
\hline $20 \mathrm{mg}$ & 94.4 \\
\hline $28 \mathrm{mg}$ & 5.6 \\
\hline \multicolumn{2}{|l|}{ Dosage (\%) } \\
\hline Fixed & 94.4 \\
\hline Flexible & 5.6 \\
\hline \multicolumn{2}{|l|}{ Regimen (\%) } \\
\hline qd & 31.6 \\
\hline bid & 68.4 \\
\hline Length (mean) [range] & 39.1 [12-208] \\
\hline 12-24weeks (\%) & 15.8 \\
\hline v $\geq 24-36$ weeks (\%) & 52.6 \\
\hline$\geq 36$ weeks (\%) & 31.6 \\
\hline \multicolumn{2}{|l|}{ Patients } \\
\hline Number of patients & 5004 \\
\hline Age (mean) [range] & 75.8 [65.2-84.6] \\
\hline$<75$ years $(\%)$ & 33.3 \\
\hline$\geq 75-77$ years (\%) & 38.9 \\
\hline
\end{tabular}

Table 1 Studies, interventions and patients' characteristics, and risk of bias of included clinical trials (Continued)

\begin{tabular}{|c|c|}
\hline \multicolumn{2}{|l|}{ Studies } \\
\hline$\geq 77-79$ years $(\%)$ & 22.2 \\
\hline$\geq 80$ years $(\%)$ & 5.6 \\
\hline Women (\%) [range] & $59.5[2.9-86.7]$ \\
\hline Baseline cognitive function (mean) ${ }^{c}[$ range $]$ & $45.6[24.3-72.4]$ \\
\hline $\begin{array}{l}\text { Baseline severity neuropsychiatric symptoms } \\
\text { (mean) }{ }^{\mathrm{C}} \text { [range] }\end{array}$ & $11.3[4.4-25.4]$ \\
\hline Baseline functional ability (mean) ${ }^{c}$ [range] & $65.7[50.2-79.6]$ \\
\hline \multicolumn{2}{|l|}{ Scales of efficacy assessment ${ }^{a}$} \\
\hline \multicolumn{2}{|l|}{ Cognitive function } \\
\hline ADAS-Cog (\%) & 35.3 \\
\hline MMSE (\%) & 41.2 \\
\hline SIB (\%) & 23.5 \\
\hline \multicolumn{2}{|l|}{ Global change } \\
\hline CIBIC-Plus (\%) & 80.0 \\
\hline CGI (\%) & 20.0 \\
\hline \multicolumn{2}{|l|}{ Neuropsychiatric symptoms } \\
\hline NPI (\%) & 100 \\
\hline \multicolumn{2}{|l|}{ Functional ability } \\
\hline 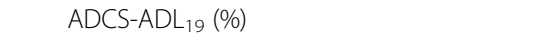 & 60.0 \\
\hline 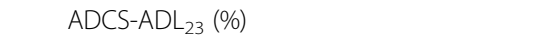 & 40.0 \\
\hline \multicolumn{2}{|l|}{ High risk of bias ${ }^{d}$} \\
\hline Discontinuation outcomes & 0 \\
\hline Efficacy cognitive function & 17.6 \\
\hline Efficacy global change & 10.0 \\
\hline Efficacy neuropsychiatric symptoms & 20.0 \\
\hline Efficacy functional ability & 10.0 \\
\hline Safety outcomes & 0 \\
\hline
\end{tabular}

Abbreviations: $A D$ Alzheimer's disease, $A D A S$-Cog Alzheimer's disease Assessment Scale-Cognitive subscale, ADCS-ADL Alzheimer's Disease Cooperative Study Activities of Daily Living Inventory 19- or 23-item scale, bid twice a day, CGI Clinical Global Impression, CIBIC-Plus Clinician Interview-Based Impression on Change-Plus Caregiver Input, ITT intention to treat, MMSE MiniMental State Examination, NPI Neuropsychiatric Inventory, SIB Severe Impairment Battery, ad once a day

${ }^{a}$ One RPCCT included with factorial design

bProportion of drug-placebo comparisons

'As a percentage of scale maxima (0-100)

dProportion of comparisons with high risk of bias for each outcome

mean of 39 weeks. It was longer in studies investigating memantine in combination with ChEI (52.5 weeks) than in monotherapy (30.5 weeks).

A total of 5004 patients were included in the study. The mean age was 75.8 years and over half $(59.5 \%)$ were women. Regarding AD severity, patients showed moderate cognitive and neuropsychiatric and functional impairment.

MMSE was the most frequently used scale to evaluate cognitive function (41.2\%), followed by ADAS-Cog (35.2\%) and SIB (23.5\%). CIBIC-Plus was the most commonly used instrument to assess global change (80\%). 
All studies analysed used NPI for neuropsychiatric symptoms, and ADCS-ADL for functional ability.

\section{Risk of bias}

None of the studies scored "high risk of bias" neither for discontinuation nor for safety outcomes. For efficacy outcomes, two studies were deemed to have a high risk of attrition bias. One of them showed differences in discontinuation rate between study groups [22]. The other study [71] had a notable discontinuation rate and performed an analysis per protocol (see Additional file 4: Figs. S1, S2 and Table S17).

\section{Meta-analysis and effect of covariates}

Table 2 shows the effect of memantine on study outcomes, and Table 3 the effect of study design-, intervention-, and patient-covariates.

\section{Discontinuation outcomes}

Regarding all-cause treatment discontinuation, 4989 patients from seventeen studies were included in the analysis. The discontinuation rate was relatively low amongst patients receiving either memantine or placebo $(18.2 \%$ vs $19.4 \%)$, and no statistically significant differences were found (OR=0.97 [0.82, 1.14], Fig. 2). The statistical heterogeneity was low $\left(I^{2}=14.9 \%\right)$ and the meta-regression analysis showed that baseline functional ability was positively associated with all-cause discontinuation ( $\log \mathrm{OR}=0.028$ [0.001, 0.055]), explaining 68\% of the variability observed (Fig. 3). For discontinuation due to $\mathrm{AE}$, no statistically significant differences were observed from placebo (14 memantine vs placebo comparisons; 4632 patients; OR=1.18 [0.91, 1.53], Fig. 4). A

Table 2 Effect of memantine on discontinuation, efficacy and safety outcomes in patients with Alzheimer's disease

\begin{tabular}{lllll}
\hline & $\mathrm{N}^{\mathrm{a}}$ & $\mathrm{OR}$ & $95 \% \mathrm{Cl}$ & $\mathrm{F}^{2}(\%)$ \\
\hline All-cause discontinuation & 18 & 0.97 & $0.82,1.14$ & 14.9 \\
Discontinuation due to AE & 14 & 1.18 & $0.91,1.53$ & 34.4 \\
Discontinuation due to LoE & 7 & 0.40 & $0.18,0.87$ & 0 \\
& $\mathrm{~N}$ & $\mathrm{SMD}$ & $95 \% \mathrm{Cl}$ & $\mathrm{P}^{2}(\%)$ \\
Cognitive function & 17 & 0.15 & $0.08,0.22$ & 24.3 \\
Global change & 10 & 0.16 & $0.08,0.24$ & 29.3 \\
Neuropsychiatric symptoms & 15 & 0.16 & $0.09,0.24$ & 27.2 \\
Functional ability & 10 & 0.07 & $-0.02,0.15$ & 14.7 \\
& $\mathrm{~N}$ & $\mathrm{OR}$ & $95 \% \mathrm{Cl}$ & $\mathrm{P}^{2}(\%)$ \\
Proportion patients AE & 6 & 1.05 & $0.88,1.25$ & 0 \\
Proportion patients SAE & 10 & 0.89 & $0.70,1.13$ & 18.3 \\
Mortality & 13 & 1.03 & $0.74,1.44$ & 0
\end{tabular}

$A E$ adverse event, $C h E I$ cholinesterase inhibitor, $\mathrm{Cl}$ confidence interval, $l^{2}$ heterogeneity, $L O E$ lack of efficacy, $N$ number of memantine-placebo comparisons, $O R$ odds ratio, SAE severe adverse event

${ }^{a}$ One study included had a factorial design moderate statistical heterogeneity was found $\left(I^{2}=34.4 \%\right)$ and the baseline functional ability was also positively correlated with this outcome ( $\log \mathrm{OR}=0.041$ [0.001, 0.081 ]), explaining $47 \%$ of the variability (Fig. 3). Memantine showed a better outcome than placebo on discontinuation due to LoE (7 memantine vs placebo comparisons; 3015 patients; $\mathrm{OR}=0.40$ [0.18, 0.87], Additional file 5: Fig. S3). Neither statistical heterogeneity nor statistically significant effect of any covariates were found (see Additional file 5: Table S18). Nevertheless, few studies provided data on this outcome.

\section{Efficacy outcomes}

Regarding efficacy, when compared to placebo, memantine showed a small improvement on cognitive function (16 memantine vs placebo comparisons) studies; 4336 patients; $\mathrm{SMD}=0.15$ [0.08, 0.22], Fig. 5), global symptomatology (10 memantine vs placebo comparisons; 4169 patients; SMD=0.16 [0.08, 0.24], Additional file 5: Fig. S4), and neuropsychiatric symptoms (14 memantine vs placebo comparisons; 5011 patients; $\mathrm{SMD}=0.16$ [0.09, 0.24], Additional file 5: Fig. S5). Since all the studies in the analysis used the NPI scale, we calculated an MD of 2.2 points. Conversely, no differences were found regarding functional ability (10 memantine vs placebo comparisons; 4067 patients; $\mathrm{SMD}=0.07$ [- 0.02, 0.15], Additional file 5: Fig. S6). For all efficacy outcomes, heterogeneity was low (cognitive function $I^{2}=24.3 \%$; global change $I^{2}=29.3 \%$; neuropsychiatric symptoms $I^{2}=27.2 \%$; and functional ability $\left.I^{2}=14.7 \%\right)$. Meta-regression analysis found that two covariates were correlated with neuropsychiatric symptom severity: baseline cognitive function and type of statistical analysis. Baseline cognitive function was negatively associated with neuropsychiatric symptoms (Diff SMD $=-0.005$ $[-0.009,-0.001]$ ) accounting for $88 \%$ of variability (Fig. 4). The studies that used a non-ITT analysis showed a larger effect size than those using an ITT approach (Diff $\mathrm{SMD}=0.089$ [0.011, 0.358]), representing $72 \%$ of the variance observed. No covariate was found to modify the effect of memantine on the remaining efficacy outcomes (Additional file 5: Table S18).

It is important to highlight that similar efficacy was found for memantine in both monotherapy and in combination with ChEI. No statistically significant differences were observed between the effect of memantine in monotherapy, or in combination with ChEI, on any efficacy outcome analysed (see Additional file 5: Table S19).

\section{Safety outcomes}

In relation to safety, no statistically significant differences were found in $\mathrm{AE}$ (6 memantine vs placebo comparisons; 2425 patients; $\mathrm{OR}=1.05$ [0.88, 1.25], Additional file 5: Fig. S7) nor in SAE outcome (10 memantine vs placebo comparisons; 3693 patients; 
Blanco-Silvente et al. BMC Geriatrics (2018) 18:168

Page 7 of 16

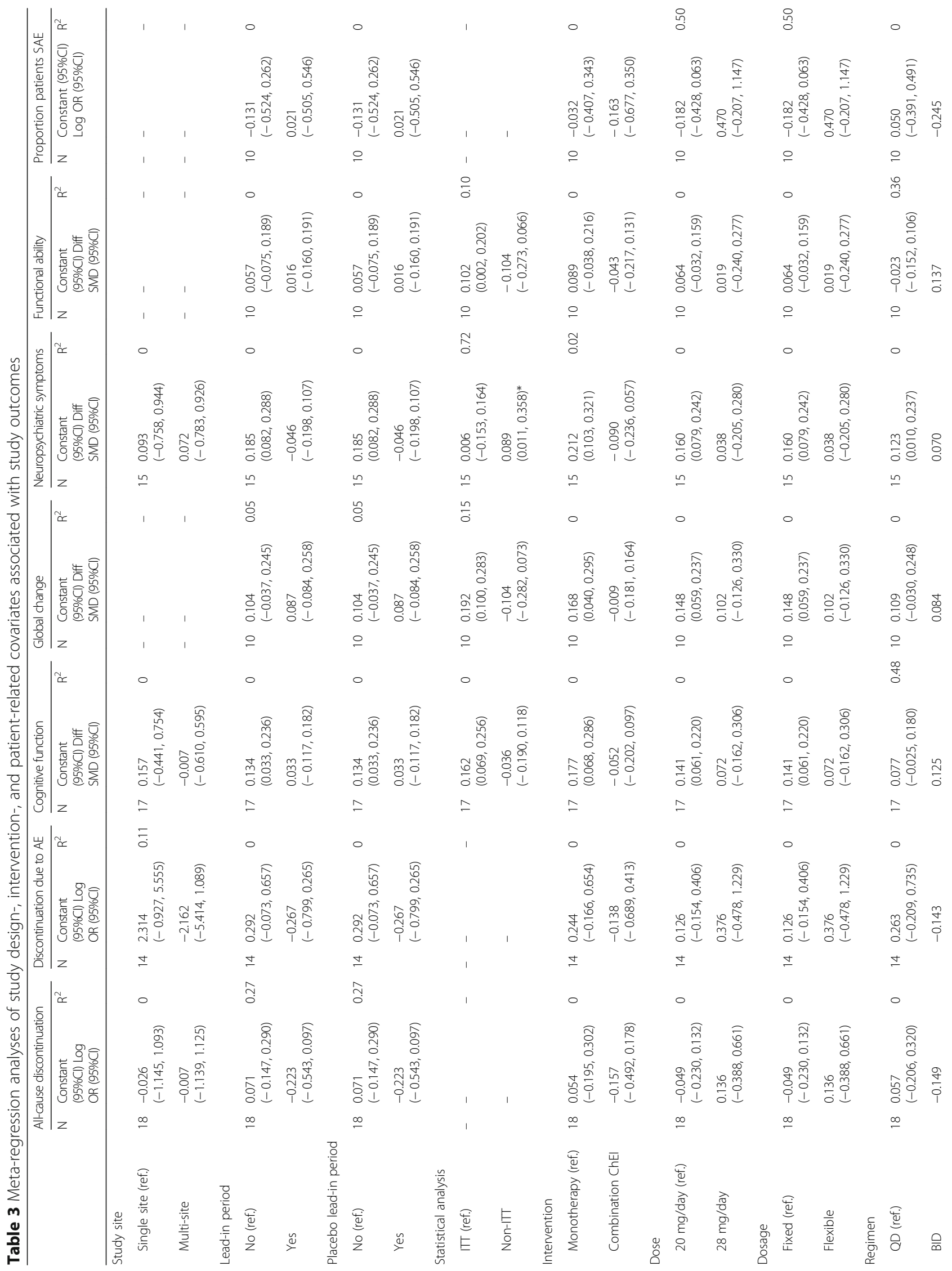


Blanco-Silvente et al. BMC Geriatrics (2018) 18:168

Page 8 of 16

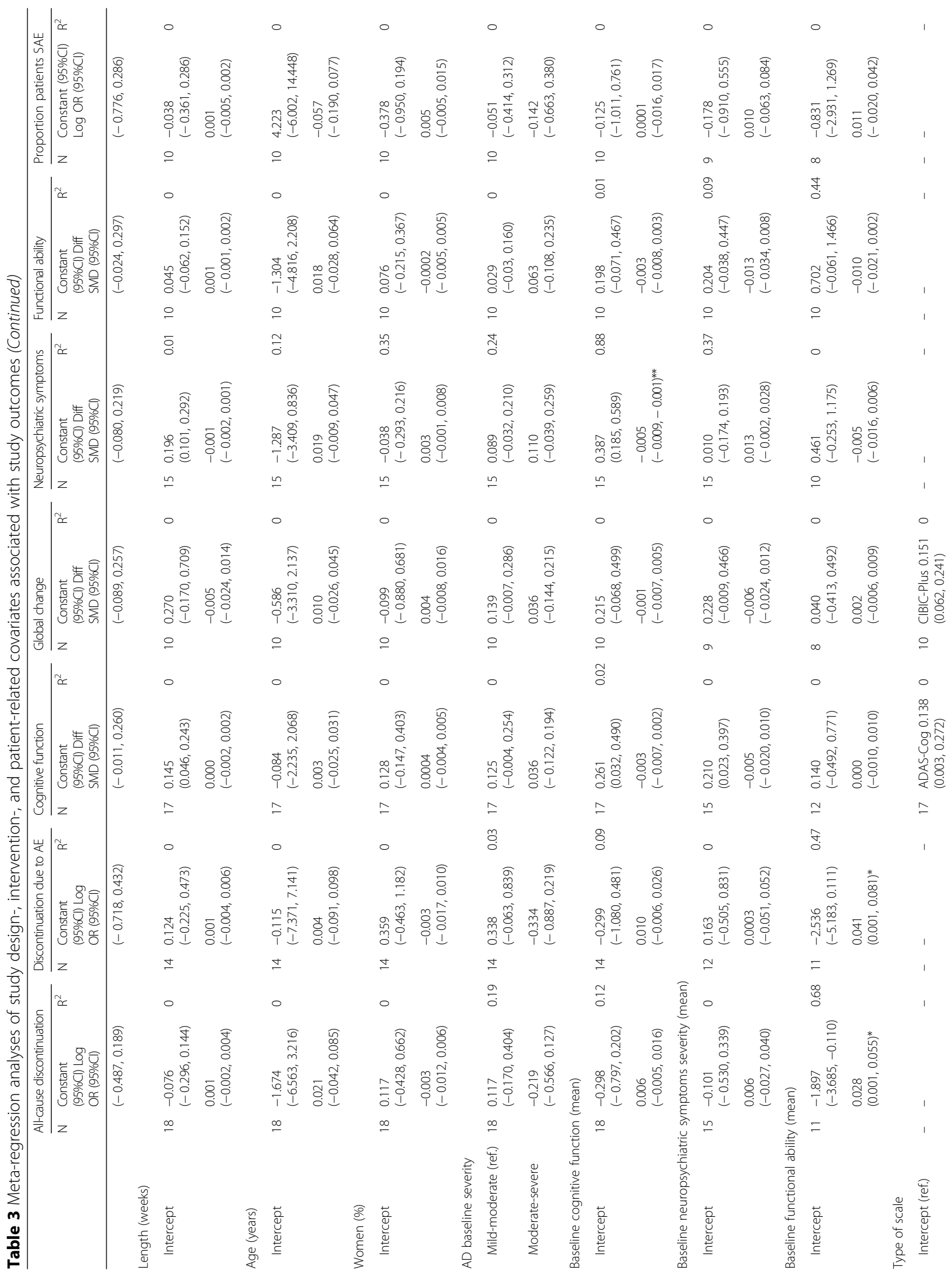




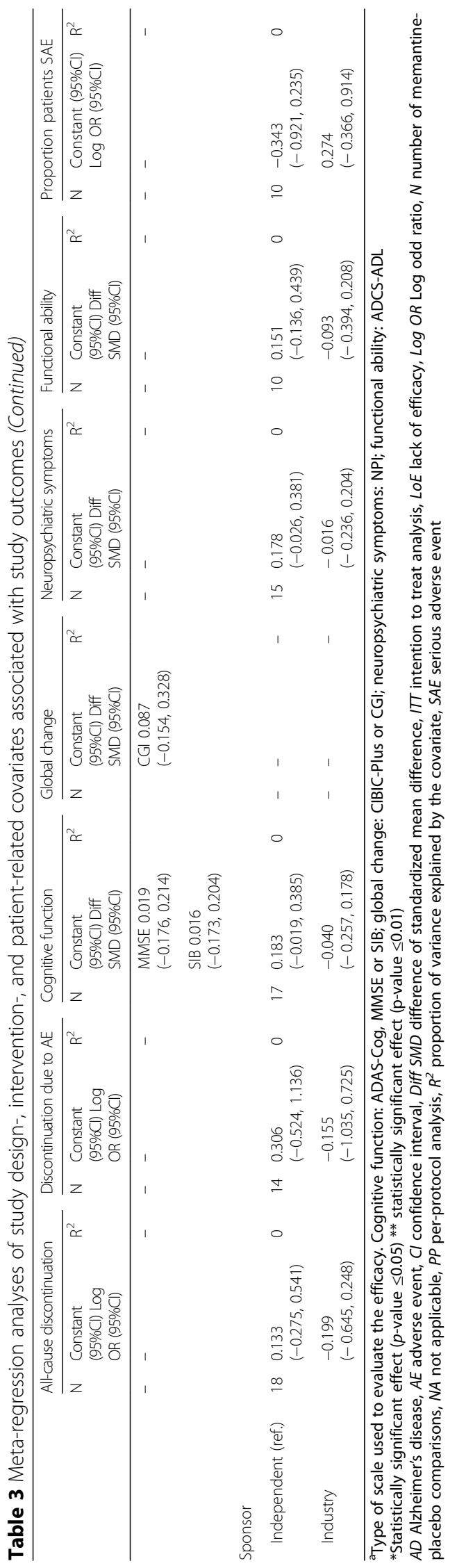




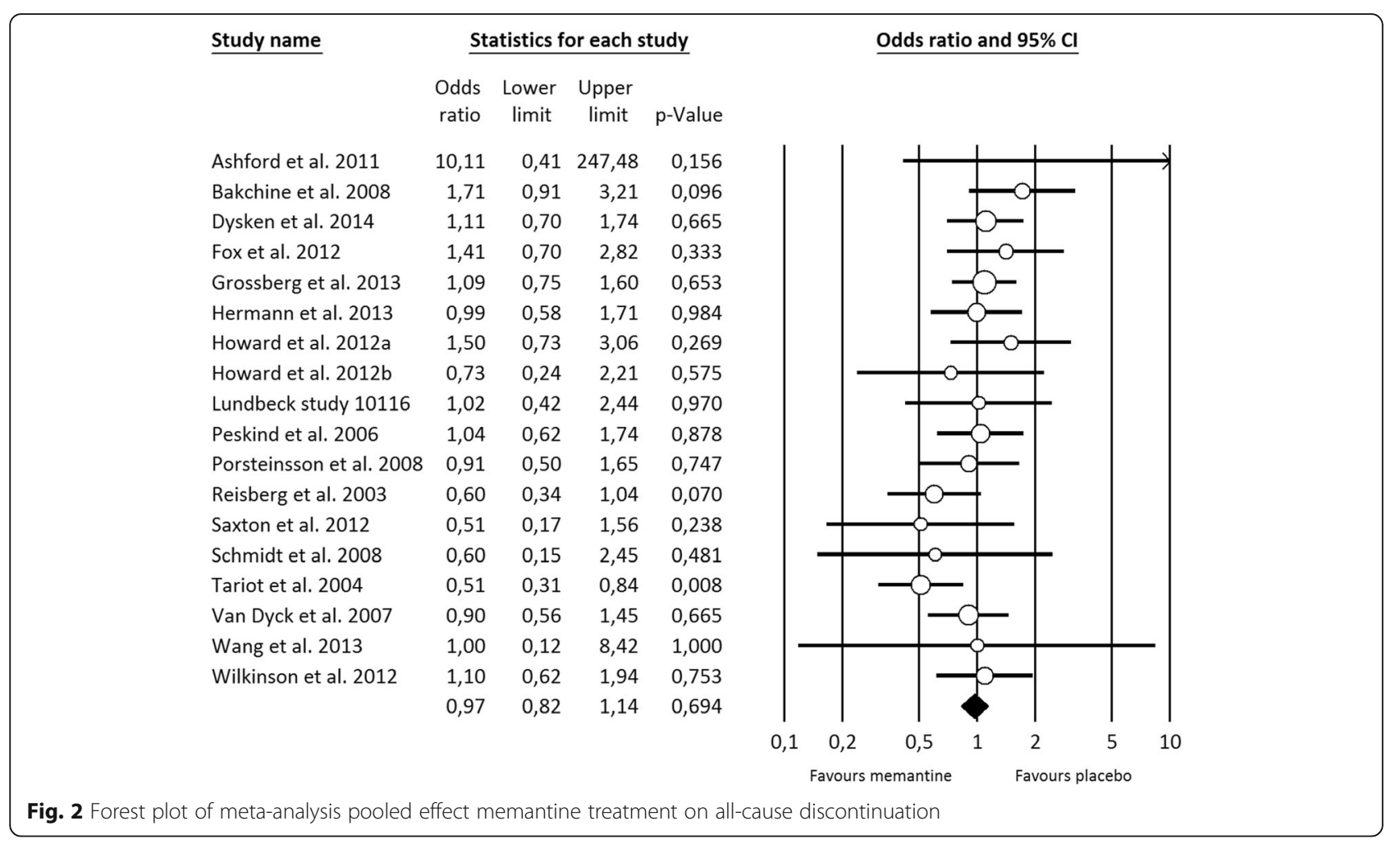

$\mathrm{OR}=0.89$ [0.70, 1.13], Additional file 5: Fig. S8), nor in mortality (12 memantine vs placebo comparisons; 4232 patients; OR=1.03 [0.74, 1.44], Additional file 5: Fig. S9). The results for DRAE were not analysed as only one study [72] provided data. Low heterogeneity was found for $\operatorname{SAE}\left(I^{2}=18.3 \%\right)$. However, no heterogeneity was observed for discontinuation due to LoE, or $\mathrm{AE}$ and mortality. No covariate had a statistically significant effect on any safety outcome (Additional file 5: Table S18).

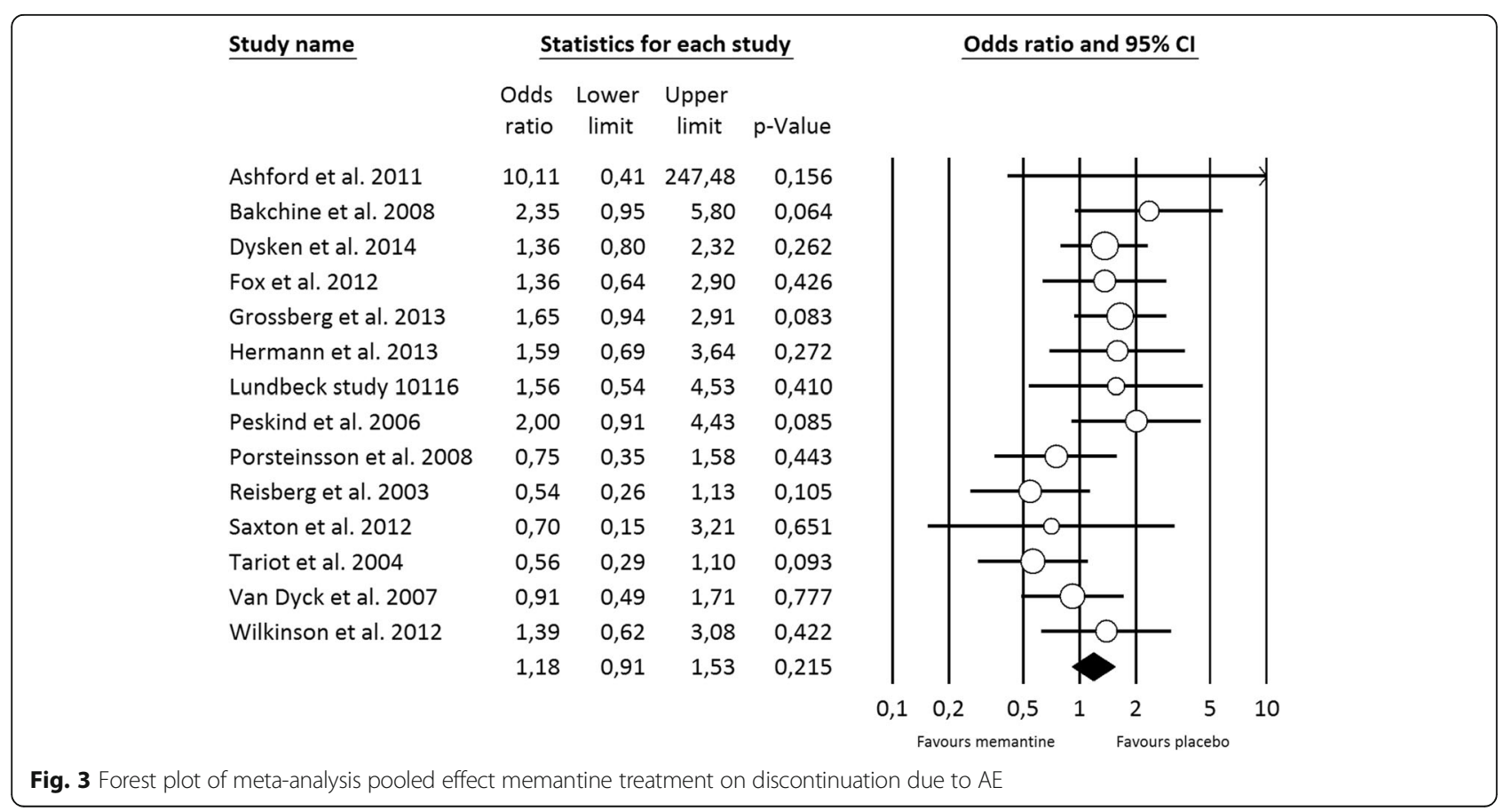




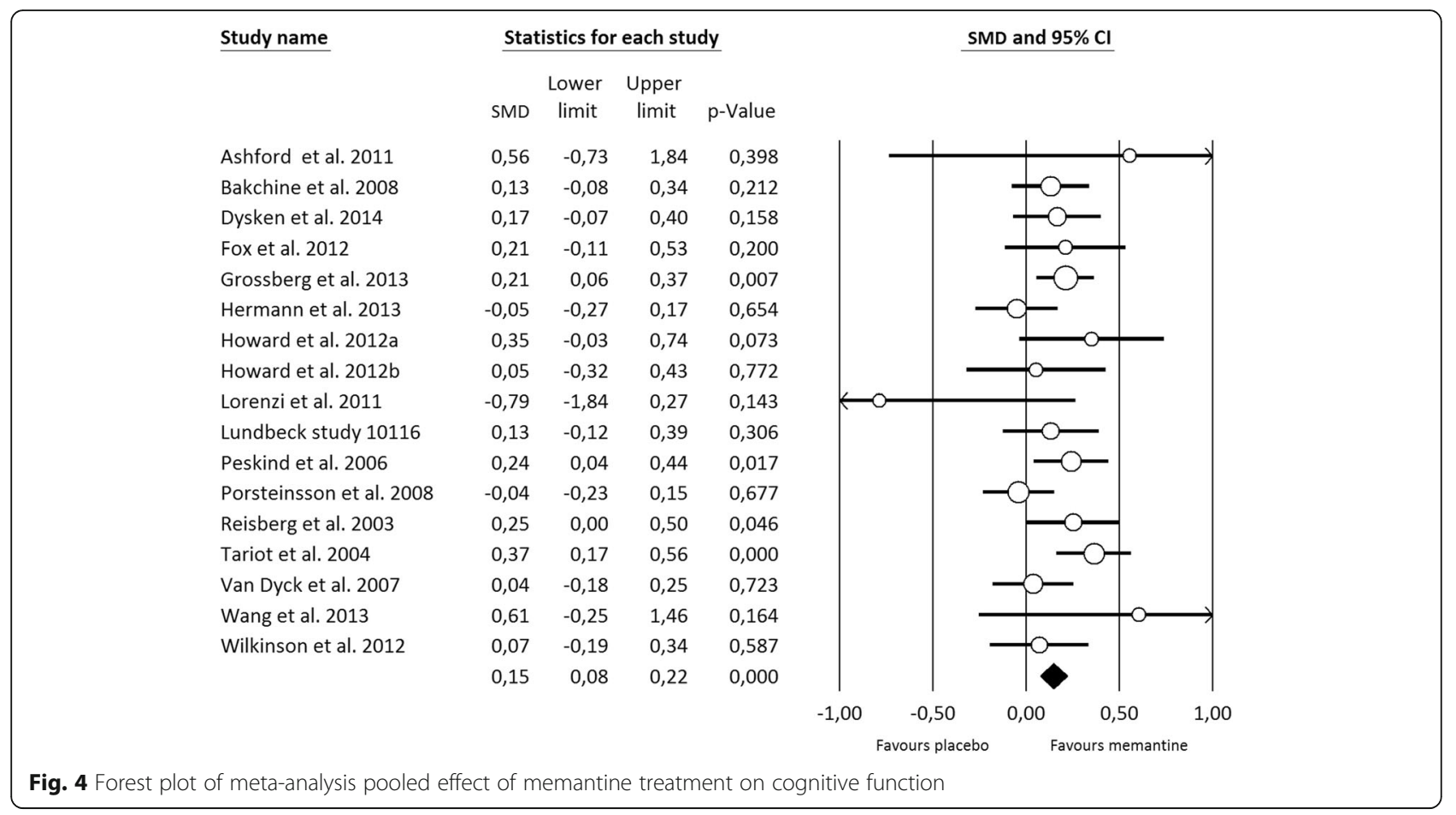

\section{Sensitivity analysis and publication bias}

Two sensitivity analyses were performed. The first excluded studies with a high risk of bias [22, 71], and the second included the results of two clinical trials identified in a pooled analysis [67] whose primary report could not be found. Both analyses found similar results to the primary analysis (see Additional file 6: Table S20 and Table S21).

No evidence of publication bias was found as none of the study outcomes showed funnel plot asymmetry. These results were confirmed with Begg's and Egger's tests (Additional file 6: Fig. S10).

\section{Discussion}

A modest number of RPCCT have investigated the efficacy of memantine for AD as monotherapy or in combination with ChEI. Most studies had an unclear methodological quality fundamentally because it is doubtful whether blinding can be guaranteed, and because the possibility of attrition bias cannot be ruled out. We have found no difference between memantine and placebo on treatment discontinuation. It must be acknowledged that this is not a favourable outcome because, ideally, any symptomatic treatment should demonstrate a lower treatment discontinuation rate than placebo, as this would indicate that the improvement of symptoms outweighs side effects. Regarding discontinuation due to $\mathrm{AE}$, no differences from placebo were observed. The statistical heterogeneity of these two outcomes of discontinuation is small-moderate, and a large proportion of between-study variance is explained by the patients' baseline functional ability, which is associated with better outcome on all-cause discontinuation and discontinuation due to AE. A possible explanation is that, since patients with lower baseline functional ability are more impaired, they may be less sensitive to $\mathrm{AE}$, or to report them less frequently, leading to lower discontinuation due to $\mathrm{AE}$ and a lower rate of all-cause treatment discontinuation.

Memantine is slightly more efficacious than placebo on cognitive function, global symptomatology, neuropsychiatric symptoms and discontinuation due to inefficacy. This finding is consistent with previous SRMA [17, 40-42, 48]. In addition, in accordance with Farrimond et al. [40], memantine does not improve functional ability.

Overall, these findings cast doubt on the clinical relevance of memantine's efficacy for AD. Our study differs from others that reach more optimistic conclusions $[17,42,45,48]$, probably because their interpretation overlooks the effect size of the intervention, which is low to very small in all efficacy domains [73].

Between-study variability of efficacy outcomes is small. Two covariates modify the effect of memantine effect on neuropsychiatric symptoms. Firstly, one related with patient characteristics; the baseline cognitive function in the following way: patients with greater cognitive function show less improvement in neuropsychiatric symptomatology. Nevertheless, it must be taken into account that this finding is of dubious clinical relevance because the effect size of memantine on neuropsychiatric outcomes is very small, and differences on baseline 

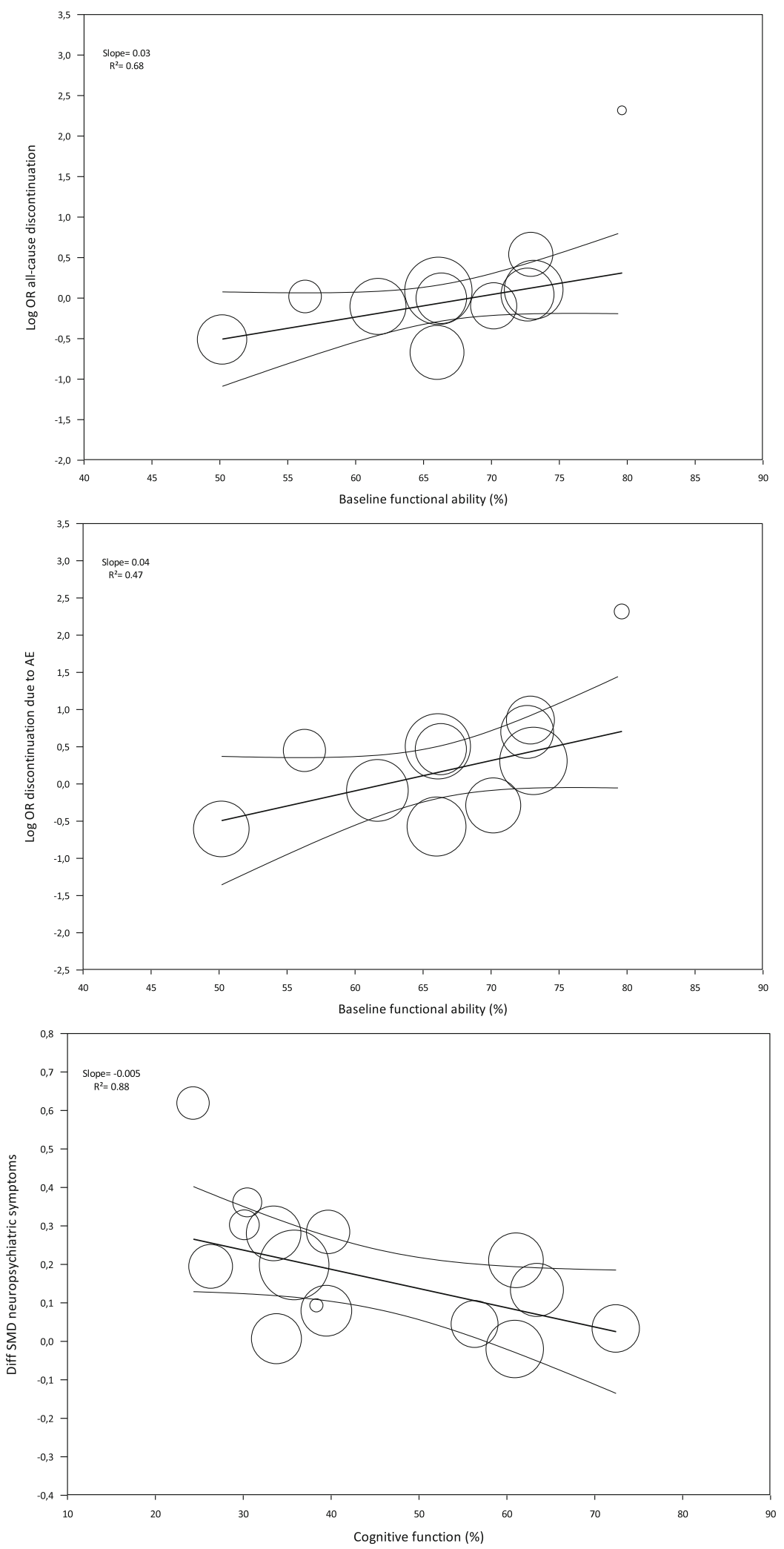

Fig. 5 Scatterplots of covariates related to study outcomes. The effect of baseline functional ability on all-cause discontinuation (Top), the effect of baseline functional ability on discontinuation due to AE (Middle) and the effect of baseline cognitive function on neuropsychiatric symptoms (Bottom) 
cognitive function might not change significantly this effect size. The second covariate that was found to modify the effect of memantine on neuropsychiatric symptoms was the type of statistical analysis; with studies using a non-ITT approach showing a larger effect size than those using an ITT approach. The overestimation of the effect size in trials deviating from an ITT approach is consistent with previous research [74]. This could be due to multiple factors such as differential exclusion of patients with severe disease or those who are not doing well in a particular study arm [75]. It is important to highlight that no intervention-related covariates modified the effect of memantine on any study outcome, indicating that the effect of memantine does not change across time, with higher doses or when it is administered in combination with ChEI.

The results of our covariate analysis contrast with those of Taro et al. [17]. In this study, the authors found that the MMSE scores at baseline and the proportion of male were associated with the efficacy on cognitive function, and the sample size and the study duration were correlated with the improvement on behavioural disturbances. In addition, they did not find an association between ITT approach and efficacy on neuropsychiatric symptoms. The differences could be explained by different reasons. Firstly, the authors performed meta-regression analyses for monotherapy and combination therapy studies separately, without taking into account if there were differences between these two subgroups. Secondly, in the study by Taro et al. [17], the baseline cognitive function was measured only with MMSE, while we also used the ADAS-cog scale. Thirdly, Taro et al. [17] included both open-label and double-blind clinical trials. The definition of the covariate "Type of statistical analysis", differed between the study by Taro et al. [17] and our study. We considered that the analysis was non-ITT when the number of patients included in the analysis was less than $95 \%$ of randomized patients, otherwise it was ITT. In contrast, Taro et al. [17] compared ITT or full analysis set population versus observed case analysis.

Regarding safety, as in previous studies [17, 41-43, 46, 48], our results support that memantine has a similar safety profile as placebo, since no differences were observed on $\mathrm{AE}, \mathrm{SAE}$ and mortality. It could be argued that patients with $\mathrm{AD}$ may underreport $\mathrm{AE}$, leading to an overestimation of memantine safety. In addition, differences in the incidence of SAE could be not detected. This was probably due to the low number of patients included and the relatively short length in clinical trials.

Overall, these results suggest that memantine has a questionable risk-benefit relationship providing a weak support for using memantine to treat patients with $\mathrm{AD}$. This contrasts with the widespread use of memantine [9-12], which can be explained by the lack of pharmacological alternatives for patients with severe $\mathrm{AD}$; a condition that is a significant burden on patients' caregivers $[3,76]$ and a considerable cost to society $[77,78]$.

\section{Limitations and strengths}

This study has limitations with regards to internal validity. The biases of RPCCT included might also bias the results of our meta-analysis. In any case, it does not appear to influence our study, as excluding the trials with a high risk of bias yields similar results to the main analyses. Publication bias can also affect results, but we found no evidence that it has affected our study. The possibility of ecological bias [79] must also be born in mind when interpreting meta-regression analyses. In addition, as patients were not randomized to the categories investigated using meta-regression, the possibility of confounding cannot be ruled out. As multiple comparisons have been made, it is possible that the differences observed have been found by chance.

In addition, there are limitations relating to external validity. The length of the studies is shorter than the current treatment with memantine in a clinical setting [80]. Furthermore, the strict inclusion criteria hinder the extrapolation of our results to clinical practice, as patients with serious comorbid diseases, which are common in clinical practice, are excluded from participating in clinical trials [81]. This is particularly relevant in relation to drug safety, thus our study may underestimate the risks associated with the administration of memantine. Finally, a further limitation affecting study precision must be taken into account when interpreting the findings of the meta-regression. This is particularly relevant to the outcomes "discontinuation due to LoE" and "proportion of patients with $\mathrm{AE}$ ", given that only a low number of studies were included in the analysis for these outcomes.

Regarding the strengths of the study, this is a comprehensive investigation of the risk-benefit of memantine for $A D$ as we have analysed several efficacy, safety and discontinuation outcomes, in addition to mortality. This is, to the best of our knowledge, the largest SRMA conducted to investigate extensively the sources of between-study heterogeneity. Our findings expand and complement the results of previous studies [17, 39-48], providing evidence of the unconvincing effect of memantine treatment in patients with AD. Furthermore, the registry in PROSPERO, the accurate quality assessment of included trials and the transparency of the data give value to our study [82].

\section{Conclusions}

This study concludes that memantine has a very small efficacy on cognitive, global and neuropsychiatric symptoms but does not improve functional ability. Despite it has a similar safety profile to that of placebo, no evidence of treatment discontinuation improvement is found, 
indicating overall that the risk-benefit relationship for the treatment of patients with $\mathrm{AD}$ is dubious. Between studyvariability is low to moderate, and no intervention characteristic or subgroup of patients clearly shows a significantly better risk-benefit relationship.

\section{Additional files}

Additional file 1: PRISMA checklist and search strategies. We provide the PRISMA checklist (Table S1) and search strategies (Table S2). (DOCX $42 \mathrm{~kb}$ )

Additional file 2: Study dataset. We provide the data used (Tables S3-S13). (DOCX 34 kb)

Additional file 3: Characteristics of included clinical trials. We provide the references of included studies (Table S14) and their study-, intervention-, and patient-related characteristics (Table S15 and Table S16), (DOCX $32 \mathrm{~kb}$ )

Additional file 4: Risk of bias of included clinical trials. We provide the risk of bias of included studies on different domains using the Cochrane Collaboration tool (Figure S2 and Figure. S3) and the high risk of bias by study outcomes (Table S17). (DOCX $73 \mathrm{~kb}$ )

Additional file 5: Forest plot of secondary outcomes and additional results. We provide the forest plot of secondary outcomes (Figure S3, Figure S4, Figure S5, Figure S6, Figure S7, Figure S8 and Figure S9), the results of meta-regression of some secondary outcomes not provided in the manuscript (Table S18) and the results of the meta-analysis of study outcomes by type of intervention (monotherapy vs. combination with ChEl) (Table S19). (DOCX $249 \mathrm{~kb}$ )

Additional file 6: Sensitivity analyses and publication bias. We provide the results of the two sensitivity analyses: by excluding studies with high risk of bias (Table S20) and by including the results of Nakamura et al. 2014 pooled analysis (Table S21); and the funnel plots of study outcomes (Figure S10). (DOCX $291 \mathrm{~kb}$ )

\section{Abbreviations}

AD: Alzheimer's disease; ADAS-cog: Alzheimer's disease Assessment ScaleCognitive subscale; ADCS-ADL: Alzheimer's Disease Cooperative Study Activities of Daily Living Inventory 19- or 23-item Scale; AE: Adverse event; BEHAVE-AD: Behavioural Pathology in Alzheimer's Disease Rating Scale; BID: Twice daily regimen; CGI: Clinical Global Impression; ChEl: Cholinesterase inhibitor; Cl: Confidence interval; CIBIC-Plus: Clinician Interview-Based Impression on Change-Plus Caregiver Input; DAD: Disability Assessment for Dementia; DRAE: Drug-related adverse event; ITT: Intention to treat analysis data; LoE: Lack of efficacy; MMSE: Mini-mental State Examination; NICE: National Institute of Health and Care Excellence; NMDA: N-methyl-Daspartate; NPI: Neuropsychiatric Inventory; OR: Odds ratio; PP: Per-protocol analysis; PRISMA: Preferred Reporting Items for Systematic Reviews and MetaAnalysis; PROSPERO: International Prospective Register of Systematic Reviews; QD: Once daily regimen; RPCCT: Randomized placebo-controlled clinical trial; SAE: Severe adverse event; SIB: Severe Impairment Battery; SMD: Standard mean difference; SRMA: Systematic review and meta-analysis

\section{Acknowledgements}

We would like to thank authors and professionals who provided the data required for the study.

\section{Funding}

This study was supported by Universitat de Girona (grant nos. IFUdG2015/17 and MPCUdG2016/ref50). The funders had no role in study design, data collection and analysis, decision to publish, or preparation of the manuscript.

\section{Availability of data and materials}

All data used have been reported in the manuscript and in the additional files.

\section{Authors' contributions}

$L B, X C$ and $D C$ are the responsible for the conceptualization and for the study design. $L B$ and $X C$ performed the collection and validation of the data, as well as the formal analysis. $L B, X C, D C$, JG and JV performed the interpretation of the data. LB and XC performed the original draft. XC, DC, JG and JV performed the critical revision of the manuscript, and all authors approved the final version of the manuscript.

\section{Ethics approval and consent to participate}

Not applicable.

\section{Consent for publication}

Not applicable.

\section{Competing interests}

The authors declare that they have no competing interests.

\section{Publisher's Note}

Springer Nature remains neutral with regard to jurisdictional claims in published maps and institutional affiliations.

\section{Author details}

${ }^{1}$ TransLab Research Group; Department of Medical Sciences, University of Girona, Girona, Spain. ${ }^{2}$ Department of Medical Sciences, Faculty of Medicine, University of Girona, Emili Grahit, 77, 17003 Girona, Spain. ${ }^{3}$ Girona Biomedical Research Institute (IdibGi), Parc Hospitalari Martí i Julià, Salt, Spain.

Received: 10 January 2018 Accepted: 9 July 2018

Published online: 24 July 2018

\section{References}

1. World Health Organization. Dementia. Geneva: WHO; 2015.

2. Jönsson $L$, Eriksdotter Jönhagen $M$, Kilander $L$, et al. Determinants of costs of care for patients with Alzheimer's disease. Int J Geriatr Psychiatry. 2006; 21(5):449-59.

3. Jones RW, Lebrec J, Kahle-Wrobleski K, et al. Disease progression in mild dementia due to Alzheimer disease in an 18-month observational study (GERAS): the impact on costs and caregiver outcomes. Dement Geriatr Cogn Disord Extra. 2017;7(1):87-100.

4. Müller WE, Mutschler E, Riederer P. Noncompetitive NMDA receptor antagonists with fast open-channel blocking kinetics and strong voltagedependency as potential therapeutic agents for Alzheimer's dementia. Pharmacopsychiatry. 1995;28(4):113-24.

5. Wenk GL, Danysz W, Mobley SL. MK-801, memantine and amantadine show neuroprotective activity in the nucleus basalis magnocellularis. Eur J Pharmacol. 1995;293(3):267-70.

6. European Medicines Agency. Ebixa memantine. London: EMA; 2002.

7. Food and Drug Administration. Drug approval package Namenda (Memantine HCl). Silver Spring: FDA; 2003.

8. Schmidt R, Hofer E, Bouwman FH, et al. EFNS-ENS/EAN guideline on concomitant use of cholinesterase inhibitors and memantine in moderate to severe Alzheimer's disease. Eur J Neurol. 2015;22(6):889-98.

9. National Institute for Health and Clinical Excellence. Donepezil, galantamine, rivastigmine and memantine for the treatment of Alzheimer's disease. London: NICE; 2011.

10. Calvó-Perxas L, Turró-Garriga O, Vilalta-Franch J, et al. Trends in the prescription and long-term utilization of antidementia drugs among patients with Alzheimer's disease in Spain: a cohort study using the registry of dementias of Girona. Drugs Aging. 2017;34(4):303-10.

11. Kadohara K, Izumi Sato I, Doi Y, et al. prescription patterns of medications for Alzheimer's disease in Japan from 2010 to 2015: a descriptive pharmacy claims database study. Neurol Ther. 2017;6(1):25-37.

12. Koller D, Hua T, Bynum J. Treatment patterns with antidementia drugs in the united sates: Meidcare cohort study. J Am Geriatr Soc. 2016;64(8): $1540-8$.

13. Taipale H, Tanskanen A, Koponen M, Tolppanen AM, Tiihonen J, Hartikainen S. Antidementia drug use among community-dwelling individuals with Alzheimer's disease in Finland: a nationwide register-based study. Int Clin Psychopharmacol. 2014;29(4):216-23. 
14. Higgins JPT, Green S. Assessing risk of bias in included trials. In: Higgins JPT, Green S, editors. Cochrane handbook for systematic reviews of interventions version 5.1.0. Copenhagen: The Cochrane Collaboration; 2011.

15. Schneider LS, Tariot PN, Dagerman KS, et al. Effectiveness of atypical antipsychotic drugs in patients with Alzheimer's disease. N Engl J Med. 2006;355(15):1525-38.

16. Blanco-Silvente L, Castells X, Saez M, et al. Discontinuation, efficacy and safety of cholinesterase inhibitors for Alzheimer's disease: a meta-analysis and meta-regression of 43 randomized clinical trials enrolling 16,106 patients. Int J Neuropsychopharmacol. 2017;20(7):519-28.

17. Taro K, Matsunga S, Oya K, Nomura I, Ikuta T. Memantine for Alzheimer's disease: an updated systematic review and meta-analysis. J Alzheimers Dis 2017;60(2):401-25.

18. Cunill $R$, Castells $X$, Tobias A, Capella D. Pharmacological treatment of attention deficit hyperactivity disorder with comorbid drug dependence. J Psychopharmacol. 2015;29(1):15-23.

19. Riera M, Castells X, Tobias A, Cunill R, Blanco L, Capellà D. Discontinuation of pharmacological treatment of children and adolescents with attention deficit hyperactivity disorder: meta-analysis of 63 studies enrolling 11,788 patients. Pyschopharmacology. 2017;234(17):2657-71.

20. Stroup TS, Mcevoy JP, Swartz MS, et al. The National Institute of Mental Health clinical antipsychotic trials of intervention effectiveness (CATIE) project: schizophrenia trial design and protocol development. Schizophr Bull. 2003;29(1):15-31.

21. Reisberg B, Doody R, Stoffler A, Schmitt F, Ferris S, Mobius HJ. Memantine in moderate-to-severe Alzheimer's disease. N Engl J Med. 2003;348:1333-41.

22. Tariot PN, Farlow MR, Grossberg GT, Graham SM, McDonald S, Gergel I. Memantine study group. Memantine treatment in patients with moderate to severe Alzheimer disease already receiving donepezil: a randomized controlled trial. JAMA. 2004;291(3):317-24.

23. Bakchine S, Loft H. Memantine treatment in patients with mild to moderate Alzheimer's disease: results of a randomized, double-blind, placebocontrolled 6-month study. J Alzheimers Dis. 2008;13(1):97-107.

24. Dysken MW, Sano M, Asthana S, et al. Effect of vitamin E and memantine on functional decline in Alzheimer disease. JAMA. 2014;311(1):33-44.

25. Grossberg GT, Manes F, Allegri RF, et al. The safety, tolerability, and efficacy of once-daily memantine (28 mg): a multinational, randomized, double-blind, placebo-controlled trial in patients with moderate-to-severe Alzheimer's disease taking cholinesterase inhibitors. CNS Drugs. 2013;27(6):469-78.

26. Herrmann N, Gauthier S, Boneva N, Lemming OM. A randomized, doubleblind, placebo-controlled trial of memantine in a behaviorally enriched sample of patients with moderate-to-severe Alzheimer's disease. Int psychogeriatrics. 2013;25(6):919-27

27. van Dyck CH, Tariot PN, Meyers B, Malca Resnick E. Memantine MEM-MD-01 study group. A 24-week randomized, controlled trial of memantine in patients with moderate-to-severe Alzheimer disease. Alzheimer Dis Assoc Disord. 2007:21(2):136-43.

28. Higgins JPT, Green S. Identifying and measuring heterogeneity. In: Higgins JPT, Green S, editors. Cochrane handbook for systematic reviews of interventions version 5.1.0. Copenhagen: The Cochrane Collaboration; 2011.

29. Guyatt GH, Oxman AD, Kunz R, et al. GRADE guidelines: 7. Rating the quality of evidence-inconsistency. J Clin Epidemiol. 2011;64(12):1294-302.

30. Cunill R, Castells X, Tobias A, Capella D. Efficacy, safety and variability in pharmacotherapy for adults with attention deficit hyperactivity disorder: a meta-analysis and meta-regression in over 9000 patients. Psychopharmacology. 2016;233(2):187-97.

31. Undurraga J, Baldessarini RJ. Randomized, placebo-controlled trials of antidepressants for acute major depression: thirty-year meta-analytic review. Neuropsychopharmacology. 2012;37(4):851-64.

32. Abdel-Shaheed C, Maher C, Williams K, Day R, McLachlan A. Efficacy, tolerability, and dose-dependent effects of opioid analgesics for low back pain. JAMMA Intern Med. 2016;176(7):958-68.

33. Castells X, Ramos-Quiroga JA, Rigau D, et al. Efficacy of methylphenidate for adults with attention-deficit hyperactivity disorder: a meta-regression analysis. CNS Drugs. 2011;25(2):157-69.

34. Leucht S, Tardy M, Komossa K, et al. Antipsychotic drugs versus placebo for relapse prevention in schizophrenia: a systematic review and meta-analysis. Lancet. 2012;379(9831):2063-71.

35. Pérez-Mañá C, Castells X, Torrens M, Capella D, Farre M. Efficacy of psychostimulant drugs for amphetamine abuse or dependence. Cochrane Database Syst Rev. 2013;9:CD009695.
36. Stone M, Laughren $\mathrm{T}$, Jones $\mathrm{ML}$, et al. Risk of suicidality in clinical trials of antidepressants in adults: analysis of proprietary data submitted to US Food and Drug Administration. BMJ. 2009;339:b2880.

37. Schwartz S, Correll CU. Efficacy and safety of atomoxetine in children and adolescents with attention-deficit/hyperactivity disorder: results from a comprehensive meta-analysis and metaregression. J Am Acad Child Adolesc Psychiatry. 2014;53(2):174-87.

38. Leucht S, Leucht C, Huhn M, et al. Sixty years of placebo-controlled antipsychotic drug trials in acute schizophrenia: systematic review, Bayesian meta-analysis, and meta-regression of efficacy predictors. Am J Psychiatry. 2017;174(10):927-42.

39. Di Santo SG, Prinelli F, Adorni F, Caltagirone C, Musicco M. A meta-analysis of the efficacy of donepezil, rivastigmine, galantamine, and memantine in relation to severity of Alzheimer's disease. J Alzheimers Dis. 2013;35(2):349-61.

40. Farrimond $L$, Roberts $E, M c S h a n e ~ R$. Memantine and cholinesterase inhibitor combination therapy for Alzheimer's disease: a systematic review. BMJ Open. 2012;2:e000917.

41. Matsunga S, Kishi T, Iwata N. Memantine monotherapy for Alzheimer's disease: a systematic review and meta-analysis. PLoS One. 2015;10(4):e0123289.

42. Matsunga S, Kishi T, Iwata N. Combination therapy with cholinesterase inhibitors and memantine for Alzheimer's disease: a systematic review and meta-analysis. Int J Neuropsychopharmacol. 2015;18(5):pyu115.

43. McShane R, Areosa Sastre A, Minakaran N. Memantine for dementia. Cochrane Database of Systematic Reviews 2006, Issue 2. Art. No.: CD003154. https://doi.org/10.1002/14651858.CD003154.pub5.

44. Muayqil T, Camicioli R. Systematic review and meta-analysis of combination therapy with cholinesterase inhibitors and memantine in Alzheimer's disease and other dementias. Dement Geriatr Cogn Disord Extra. 2012;2(1):546-72.

45. Tan CC, Yu JT, Wang HF, et al. Efficacy and safety of donepezil, galantamine, rivastigmine, and memantine for the treatment of Alzheimer's disease: a systematic review and meta-analysis. J Alzheimers Dis. 2014;41(2):615-31.

46. Tsoi KK, Chan JY, Leung NW, Hirai HW, Wong SY, Kwok TC. Combination therapy showed limited superiority over monotherapy for Alzheimer disease: a meta-analysis of 14 randomized trials. J Am Med Dir Assoc. 2016;17(9):863. e1-8

47. Wang J, Yu JT, Wang HF, et al. Pharmacological treatment of neurpsychiatric symptoms in Alzheimer's disease: a systematic review and meta-analysis. J Neurol Neurosurg Psychiatry. 2015;86(1):101-9.

48. Winblad B, Jones RW, Wirth Y, Stoffler A, Mobius HJ. Memantine in moderate to severe Alzheimer's disease: a meta-analysis of randomized clinical trials. Dement Geriatr Cogn Disord. 2007;24(1):20-7.

49. Moher D, Liberati A, Tetzlaff J, Altman DG. The PRISMA group. 2009 Preferred reporting items for systematic reviews and meta-analyses: the PRISMA statement. J Clin Epidemiol. 2009;62(10):1006-12.

50. Rosen WG, Mohs RC. Davis KL. A new rating scale for Alzheimer's disease. Am J Psychiatry. 1984;141(11):1356-64.

51. Folstein MF, Folstein SE, McHugh PR. (1975) "mini-mental state". A practical method for grading the cognitive state of patients for the clinician. J Psychiatr Res. 1975;12(3):189-98.

52. Saxton J, Swihart AA. Neuropsychological assessment of the severely impaired elderly patient. Clin Geriatr Med. 1989;5(3):531-43.

53. Schneider LS, Olin JT, Doody RS, Clark CM, Morris JC, Reisberg B, Schmitt FA, Grundman M, Thomas RG, Ferris SH. Validity and reliability of the Alzheimer's disease cooperative study-clinical global impression of change. The Alzheimer's disease cooperative. Alzheimer Dis Assoc Disord. 1997; 11 (Suppl 2):S22-32.

54. Guy W. CGI Clinical Global Impressions. In: ECDEU assessment manual for psychopharmacology (Department of Health, Education and Welfare). National Institute of Mental Health: Rockville; 1976.

55. Cummings JL, Mega M, Gray K, Rosenberg-Thompson S, Carusi DA, Gornbein J. The neuropsychiatric inventory: comprehensive assessment of psychopathology in dementia. Neurology. 1994;44:2308-14.

56. Reisberg B, Borenstein J, Salob SP, Ferris SH, Franssen E, Georgotas A. Behavioral symptoms in Alzheimer's disease: phenomenology and treatment. J Clin Psychiatry. 1987:48(Suppl):9-15

57. Galasko D, Bennett D, Sano M, Ernesto C, Thomas R, Grundman M, Ferris S. An inventory to assess activities of daily living for clinical trials in Alzheimer's disease. The Alzheimer's disease cooperative study. Alzheimer Dis Assoc Disord. 1997;11(Suppl 2):S33-9. 
58. Gelinas I, Gauthier L, McIntyre M, Gauthier S. Development of a functional measure for persons with Alzheimer's disease: the disability assessment for dementia. Am J Occup Therep. 1999;53(5):471-81.

59. Da Costa BR, Nüesch E, Rutjes AW, et al. Combining follow-up and change data is valid in meta-analyses of continuous outcomes: a metaepidemiological study. J Clin Epidemiol. 2013;66(8):847-55.

60. Higgins JPT, Green S. How to include multiple groups from one study. In: Higgins JPT, Green S, editors. Cochrane handbook for systematic reviews of interventions version 5.1.0. Copenhagen: The Cochrane Collaboration; 2011.

61. Cohen J. Statistical power analysis in the behavioural sciences. 2nd ed Lawrence Erlbaum Associated: Hillsdale; 1988.

62. Thorlund K, Imberger G, Johnston BC. Evolution of heterogeneity $\left(I^{2}\right)$ estimates and their $95 \%$ confidence intervals in large meta-analyses. PLoS One. 2012;7(7):e39471.

63. DerSimonian R, Laird N. Meta-analysis in clinical trials. Control Clin Trials. 1986;7(3):177-88.

64. Thompson SG, Higgins JPT. How should meta-regression analyses be undertaken and interpreted? Stat Med. 2002;21(11):1559-73.

65. Boresnstein M. Introduction to meta-analysis. United Kingdom: Wiley; 2009.

66. Borenstein $M$, Hedges L, Higgins J, Rothstein H. Comprehensive metaanalysis version 3.3.070. Biostat: Englewood; 2014.

67. Nakamura Y, Kitamura S, Homma A, Shiosakai K, Matsui D. Efficacy and safety of memantine in patients with moderate-tosevere Alzheimer's disease: results of a pooled analysis of two randomized, double-blind, placebo-controlled trials in Japan. Expert Opin Pharmacother. 2014;15(7): 913-25.

68. Sterne JA, Egger M. Funnel plots for detecting bias in meta-analysis: guidelines on choice of axis. J Clin Epidemiol. 2001;54(10):1046-55.

69. Begg CB, Mazumdar M. Operating characteristics of a rank correlation test for publication bias. Biometrics. 1994;50(4):1088-101.

70. Egger M, Davey Smith G, Schneider M, Minder C. Bias in meta-analysis detected by a simple, graphical test. BMJ. 1997;315(7109):629-34.

71. Howard R, McShane R, Lindesay J. Donepezil and memantine for moderateto-severe Alzheimer's disease. N Engl J Med. 2012;366(10):893-03.

72. Wilkinson D, Fox NC, Barkhof F, Phul R, Lemming O, Scheltens P. Memantine and brain atrophy in alzheimer's disease: a 1-year randomized controlled trial. J Alzheimers Dis. 2012;29(2):459-69.

73. Jakobsen JC, Gluud C, Winkel P, Lange T, Wetterslev J. Thresholds for statistical and clinical significance in systematic reviews with meta-analytic methods. BMC Med Res Methodol. 2014;14:120.

74. Abraha I, Cherubini A, Cozzolino F, et al. Deviation from intention to treat analysis in randomized trials and treatment effect estimates: metaepidemiological study. BMJ. 2015;350:h2445.

75. Ranganathan P, Pramesh CS, Aggarwal R. Common pitfalls in statistica analysis: intention-to-treat versus per protocol analysis. Prespect Clin Res. 2016;7(3):144-6

76. World Health Organization and Alzheimer's disease International. Dementia: a public health priority. Geneva: WHO; 2012.

77. Saint-Laurent T, Özer Stillman I, Chen S, et al. Cost-utility analysis of memantine extended release added to cholinesterase inhibitors compared to cholinesterase inhibitor monotherapy for the treatment of moderate-to-severe dementia of the Alzheimer's type in the US. J Med Econ. 2015;18(11):930-43.

78. Wimo A, Reed CC, Richard Dodelc R, et al. The GERAS study: a prospective observational study of costs and resource use in community dwellers with Alzheimer's disease in three European countries - study design and baseline findings. J Alzheimers Dis. 2013;36(2):385-99.

79. Berlin JA, Santanna J, Schmid CH, Szczech LA, Feldman HI. Anti-lymphocyte antibody induction therapy study group. Individual patient- versus grouplevel data meta-regressions for the investigation of treatment effect modifiers: ecological bias rears its ugly head. Stat Med. 2002;21(3):371-87.

80. Bent-Ennakhil N, Coste F, Xie L, et al. A real-world analysis of treatment patterns for cholinesterase inhibitors and memantine among newlydiagnosed Alzheimer's disease patients. Neurol Ther. 2017;6(1):131-44.

81. Leinonen A, Koponen M, Hartikainen S. Systematic review: representativeness of participants in RCTs of acetylchonesterase inhibitors. PLoS One. 2015;10(5):e0124500.

82. Allers K, Hoffmann F, Mathes T, Piper D. Systematic reviews with published protocols compared to those without: more effort, older search. Journal of Clin Epidemiol. 2018 Mar;95:102-10. https://doi.org/10.1016/j.jclinepi.2017.12.005.

Ready to submit your research? Choose BMC and benefit from:

- fast, convenient online submission

- thorough peer review by experienced researchers in your field

- rapid publication on acceptance

- support for research data, including large and complex data types

- gold Open Access which fosters wider collaboration and increased citations

- maximum visibility for your research: over $100 \mathrm{M}$ website views per year

At BMC, research is always in progress.

Learn more biomedcentral.com/submissions 